\section{Ferroelectricity origins}

SIR - Bussmann-Holder and Büttner ${ }^{1}$ make two remarks about my work ${ }^{2}$ on the origin of ferroelectricity in perovskite oxides: (1) that the results of self-consistent electronic structure calculations are not unique and neglect dynamical properties; and (2) that the venerable shell model $^{3}$, in which one fits numerous empirical parameters to experimental data, is sufficient to understand ferroelectrics.

The only input to modern electronic structure computations such as those I presented $^{2}$ are the nuclear charges and the positions of the nuclei, the latter of which are varied to map out the dynamical potential surface. The computations use no adjustable parameters, and thus are unique. In principle, such frozen phonon calculations allow the computation of any desired harmonic or anharmonic dynamical property, though owing to tremendous computational demands, I presented only the potential surface for the ferroelectric soft mode for nuclear displacements with a periodicity of the primitive cubic perovskite cell ${ }^{2}$. The finite temperature dynamics is not neglected but is investigated by parametrizing the potential surface obtained from first-principles calculations $^{4,5}$ rather than by experiment. The dynamics of the phase transition was not the subject of my paper ${ }^{2}$, which was about the origin of the ferroelectric instability.

The computations show incontrovertibly that the ferroelectric instability is not due to shell-model-like polarizability of the oxygen ion as advocated by Bussmann-Holder and Büttner. The shell model is an empirical model in which parameters are fitted to experiment, and there is no guarantee, as with any empirical model, that the fitting parameters have any physical meaning, or that the model correctly describes the underlying physics behind material behaviour. The present computations clearly show the importance of covalency in driving the ferroelectric instability, and illustrate the advantages of firstprinciples methods compared with parametrized models.

In the 30 years since the shell model was proposed, there have been many attempts to model ferroelectrics and other phase transitions. Although im-

\footnotetext{
1. Bussmann-Holder, A. \& Büttner, H. Nature 360,541 (1992)

2. Cohen, R. E. Nature 358, 136 (1992).

3. Dick, B. G. \& Overhauser, A. W. Phys. Rev. 112, 90 (1958).

4. Tsuneyuki, S., Tsukada, M., Aoki, H. \& Matsui, Y. Phys.

Rev. Lett. 61, 869 (1988).

5. Gong, Z. \& Cohen, R. E. Ferroelectrics 136, 113 (1992).
}

pressive agreement with experiment is sometimes obtained by introducing sufficient parameters, the ability to predict unknown properties of materials, or to predict properties of new materials, has not been demonstrated. First-principles approaches now give us the ability to predict and study new materials and to understand the underlying physical processes that govern material behaviour.

Ronald E. Cohen

Geophysical Laboratory,

Carnegie Institution of Washington,

Washington DC 20015, USA

\section{An anti-prion protein?}

SIR - The physiological function of the prion protein (PrP) is unknown, but there is strong evidence that the infectious agent of transmissible spongiform encephalopathies (the prion) consists at least in part of $\mathrm{PrP}^{\mathrm{Sc}}$, a modified form of the normally occurring cellular $\operatorname{PrP}$ $\left(\mathrm{PrP}^{\mathrm{C}}\right)^{1}$. Both forms of PrP are encoded in a single exon of the PrP gene $e^{2,3}$. Goldgaber in his Scientific Correspondence 4 noted a large open reading frame (ORF) on the antisense strand of the coding regions of mammalian PrP genes. Several genetic traits influencing spongiform encephalopathies in humans and animals have been linked to the PrP gene $^{1}$. If an 'anti-PrP' existed it could be responsible for the properties formerly ascribed to the prion protein ${ }^{4}$. In support of this hypothesis, Hewinson and colleagues detected a 4.5-kilobase RNA in bovine brain which hybridized to $\operatorname{PrP}$ sense riboprobes, and which was thus expected to represent a $\operatorname{PrP}$ antisense transcript ${ }^{5}$. We have found a similar $4.5-\mathrm{kb}$ mRNA in normal and scrapieinfected hamsters and in mice; however, our results show that this presumed 'anti-prion protein' RNA is not derived from the antisense strand of the PrP gene.

We used the sequence of the second exon of the hamster PrP gene to generate sense riboprobes (Fig. 1a). Riboprobe A detected a $4.5-\mathrm{kb}$ 'antisense' RNA in brains of normal and scrapieinfected hamsters at approximately the same level, as judged by northern analysis (Fig. 1b). The 4.5-kb RNA was polyadenylated and was not found in tissues other than brain (Fig. 1b). The fact that the $4.5-\mathrm{kb}$ RNA was detected under stringent hybridization conditions initially suggested that the 'anti-PrP' RNA was transcribed from the opposite strand of the hamster PrP gene.

If indeed the $4.5-\mathrm{kb}$ RNA were an antisense transcript of the PrP gene, then it should be absent or at least altered in mice homozygous for partially

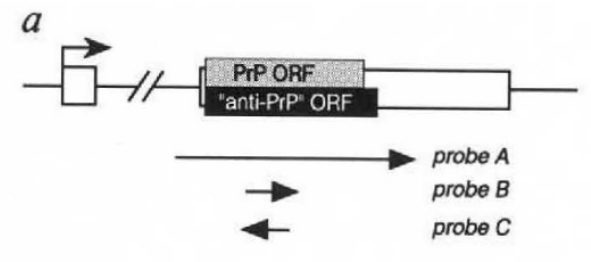

$b$ probe A

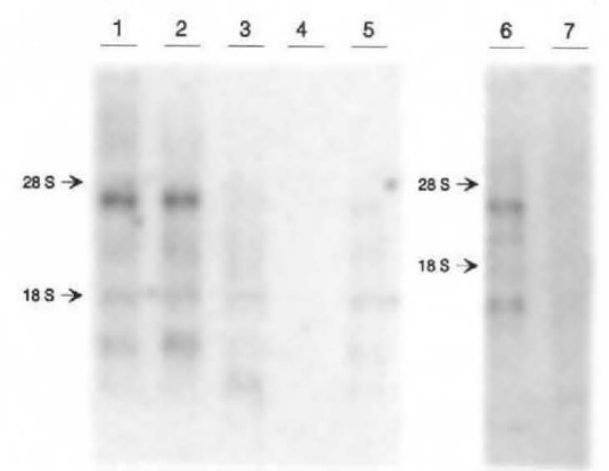

FIG. 1a, Genomic map of the hamster PrP gene $^{3}$. Digoxigenin-UTP-labelled riboprobes $\mathrm{A}-\mathrm{C}$ were generated in vitro; arrows denote direction of transcription. Probe A corresponds to the EcoRI to Dral; probe B to the Ncol-Hincil fragment of the hamster PrP gene $^{3}$; probe $\mathrm{C}$ corresponds to the $\mathrm{Kpnl}-$ BstEll fragment of mouse PrP (ref. 11). b, Northern blots of total brain RNA from normal (lane 1) or scrapie-infected (lane 2) hamsters. Lanes 3-5 show spleen, liver and lung RNA, respectively, from normal hamsters. The 4.5-kb RNA is specifically detected in polyadenylated (lane 6) but not in nonpolyadenylated (lane 7) RNA from normal hamster brain. Each lane contains $4 \mu \mathrm{g}$ total RNA, $4 \mu \mathrm{g}$ non-polyadenylated RNA or $0.4 \mu \mathrm{g}$ polyadenylated RNA. Hybridization was done with 50-100 ng probe per ml in $50 \%$ formamide, $5 x$ SSC, $2 \%$ blocking reagent (Boehringer Mannheim), $0.1 \%$ $\mathrm{N}$-lauroylsarcosine, $0.02 \%$ SDS overnight at $68{ }^{\circ} \mathrm{C}$. Hybridized probes were visualized using chemiluminescence. Exposure times: lanes 1-5, $25 \mathrm{~min}$; lanes 6,7, $15 \mathrm{~min}$.

deleted PrP genes $\left(\operatorname{Prn}-\mathrm{p}^{0 / 0}\right)^{6}$. Using the above hamster probe $A$, which was shown to cross-hybridize to 'anti-PrP' RNA from wild-type mouse brain (Fig. 2 , lanes 1 and 2 ), we detected the $4.5-\mathrm{kb}$ RNA also in mice with Prn-p $p^{0 / 0}$ genes (Fig. 2, lane 3). Because probe A extended beyond the partial deletion in the PrP gene of $\mathrm{PrP}^{0 /}$ mice, we used an additional probe which lies entirely within the deleted region (probe B). It detected the same 4.5-kb RNA in both types of mice (Fig. 2, lanes 4 and 5). These results unequivocally show that the 4.5-kb RNA is not derived from the opposite strand of the murine PrP gene; however, it appears to share considerable homology with the antisense strand of the PrP gene.

Our results confirm the presence of the 4.5-kb 'anti-PrP' RNA in two additional species, show that its concentra- 